\title{
Comparative characteristics of myohistogenesis of musculoskeletal tissue in hens and quails
}

\author{
G.R. Shakirova, E.N. Borkhunova, G.V. Kondratov, and V.V. Stepanishin* \\ Federal State Budgetary Educational Institution of Higher Education "Moscow State Academy of \\ Veterinary Medicine and Biotechnology - MVA by K.I. Skryabin", Department of Animal Anatomy \\ and Histology named after Professor A.F. Klimov, Moscow, Russia
}

\begin{abstract}
A comprehensive comparative microscopic and morphometric study of the superficial pectoral (SPM) and quadriceps femoral (QFM) muscles in hens and quails in the early stages after hatching was performed. In the muscles, diameter of the muscle fiber, area of the muscle tissue, representation of the muscle and connective tissue components were determined. A set of general regularities and specific features of postembryonic myogenesis of the studied structures in hens and quails has been established. The peculiarities of SPM and QFM ultrastructure in quails of the Manchzhurskaya zolotistaya (Manchurian gold) breed were revealed. Representatives of both studied groups of birds are characterized by an increase in the muscle tissue area, the diameter of muscle fibers and their bundles. The difference is a more significant development of the quadriceps femoris in hens, and the superficial pectoral muscle in quails. It was found that quails, unlike hens, had thickening of the endomysium and perimysium in both muscles.
\end{abstract}

\section{Introduction}

Poultry farming is an actively developing branch of agriculture. At the same time, much attention is paid to the breeding of meat and egg breeds of not only hens and turkeys, but also quails.

The quail farming industry is a promising direction in agriculture, due to the fact that this type of poultry is characterized by a set of important criteria for productivity and profitability of cultivation technology [1,9]. First of all, this concerns the speed of growth processes, which are 5 times higher than similar indicators in broiler chickens, while it is worth noting that the earlier period of the egg laying onset. Moreover, the high nutritional value of eggs and meat of these birds is well known.

The study of the development of quail skeletal muscles is of particular practical interest $[7,8,11,12]$. Skeletal musculature is represented by muscle fibers, which are characterized by a number of distinctive features, one of which is the type of energy exchange. Muscle

\footnotetext{
${ }^{*}$ Corresponding autor: stepanishin.victor@yandex.ru
} 
fibers are divided into oxidative or red fibers, and glycolytic or white muscle fibers, which can be identified by selective staining [5].

In the available literature, there is insufficient information concerning the morphological features of the muscular system of quails and hens [7, 8, 12]. All this determines the relevance of research.

The study of muscles structure in birds and mammals is covered in a large number of scientific papers [3-8, 11-14, 17, 19]. According to macro- and microscopic data, the internal structure of the myogaster of different muscles differs in terms of the ratio of muscle and connective tissue, so they have different functional characteristics. They are divided into static muscles, which have completely lost their muscle fibers and become ligamentous muscles; static-dynamic muscles with a predominance of static elements, which have largely lost their muscle fibers; static-dynamic muscles with a predominance of dynamic elements; dynamic muscles with a predominant development of the muscle part and a weakly represented tendon. When studying the innervation of the muscles on the thoracic limbs in various animals, Yu.F. Yudichev found that the thickness of nerve branches to muscles depends on the degree of muscle part development; with a decrease in their muscle mass, the thickness of the nerve branches decreases in which the latter branch out [13].

According to the Russian histologist A.A. Zavarzin (1952), the development of skeletal muscles is a restructuring of components from the cellular level of organization to the symplast, which represents the highest stage of differentiation of a certain histological structure.

R.K. Danilov in his studies showed that the nodal points of muscle tissue development are: at the cellular level, the choice of the path of differentiation by promyoblasts; tissue level - the development of myotubules and their partial death, the appearance of myosatelitocytes; organ level - the stage of myotubules myohistogenesis, the formation of neuromuscular connections, the formation of the vascular system, which determines the organization nature of energy apparatus of myotubes [4]. At the same time, the higher the considered level of muscular system organization, the more complex and diverse are the forms of tissue components interaction that make up the organ. In the development of skeletal muscle tissue, the formation of myosatellitocytes with cambial properties occurs, due to which the resistance of skeletal muscle tissue to unfavorable conditions for the body development and regeneration when it is damaged is ensured.

From the stage of late myotubules and immature muscle fibers the structuring of the contractile apparatus of the fiber comes with a pronounced ordering of thick and thin myofilaments $[18,20]$.

According to ultrastructural studies of M.D. Schmerling et al., the most important criterion for determining the variety of fiber types is the quantitative and qualitative characteristics of the mitochondrial apparatus distribution [14]. In red-type fibers, the relative representation of mitochondria exceeds that of white-type fibers [15].

The literature data indicate that the distribution of mitochondria in different types of muscle fibers is heterogeneous. Thus, in the diaphragmatic muscle fibers of the red type, under the sarcolemma, there are grouped mitochondria in the central areas, while in the white type fibers, such an organization of mitochondria is extremely rare [16].

In the results of a number of studies, it was noted that the pectoral and pelvic muscles are characterized by different representation of muscle fibers. White fibers occupy from 70 to $98 \%$ of the representation in the pectoral muscles in hens, while the pelvic limb muscles contain a greater number of red-type muscle fibers [2].

The results of studying the features of the structural design of skeletal muscles, as the most promising in the consumer aspect, were reflected in a number of our works $[7,8]$. It is worth noting that the studies present key aspects of the skeletal muscle tissue 
morphogenesis and its micromorphological parameters. At the same time, such works devoted to the study of quails in this matter are extremely few, which reflects the relevance of considering the features of myogenesis and micromorphometric indicators of skeletal muscles in ontogenesis in this type of poultry, as well as conducting a comparative analysis according to the methodological scheme proposed by us $[7,8,12]$.

The purpose of the work is to present comparative morphological and morphometric data on the structure of the quadriceps femoris and the superficial pectoral muscle in hens and quails of meat and egg breeds.

\section{Materials and Methods}

The work was carried out on the basis of the Department of Animal Anatomy and Histology named after Professor A.F. Klimov Moscow SAVMB and on the basis of the JSC Technology Department of the FSC ARRTPI RAS.

The object of the study was quail chickens of the Manchurian golden breed and chickens of hen of Smena 8 "Plymouthrock" cross.

Samples of the superficial pectoral muscle (hereinafter - SPM) and the straight head of the quadriceps femoris (hereinafter - QFM) were taken from quails on the 1st, 8th, 21st, 35 th, 42nd day after hatching, from hen chickens on the 1st, 8th, 20th, 29th, and 50th day after hatching. In each age group, 6 individuals were examined.

The material was fixed in formalin, followed by filling in paraffin according to generally accepted methods. Histological sections were made on a rotary automated microtome HM-325 (Microm international GmbH, Germany). The resulting material was stained with hematoxylin and eosin, according to van Gieson and Mallory. Histological sections and microphotography were studied using a Jenamed 2 light microscope (Carl Zeiss, Jena, Germany) combined with the ImageScope $\mathrm{C}$ digital microscopy system (Systems for Microscopy and Analysis, LLC). In hen chickens and quails, morphometric studies of muscle fibers were performed in terms of thickness, their bundles, endomysium, perimysium, and the ratio of muscle and connective tissues at all stages of myohistogenesis in SPM and QFM.

For an in-depth study of the quail muscles structure, ultrastructural studies of SPM and QFM samples were performed on the 50th day of development. During electronic microscopic examination, the tissue samples were fixed in $2.5 \%$ glutaraldehyde $(\mathrm{pH} 7.2$ 7.4 ), followed by additional fixation in $1 \%$ osmium solution on the corresponding buffer. The material was poured into Epon 812. Ultrathin sections were prepared on the LKB - 3 ultratome (Sweden), contrasted with a $2 \%$ aqueous solution of uranyl acetate, lead citrate according to Reynolds (Weekley B., 1975) and studied on a JEM CX 2 transmission microscope (JEOL Ltd., Japan) at magnifications from 4000 to 35000.

\section{Results and discussion}

\subsection{Histological and morphometric characteristics of skeletal muscles of hens of Smena 8 "Plymouthrock" cross}

When studying the age-related structural and micromorphometric features of the muscles in hens, it was found that their structure was formed at the time of hatching, differing from that of adult individuals by morphometric indicators and some morphological features of muscle fibers. Thus, the muscle fibers of a day-old chicken have a transverse striation, but are very thin, and the nuclei in the myosymplast are located both in the center and on the periphery, which indicates insufficient development of the contractile apparatus. At the 
same time, the nuclei are large, light, and numerous, which indicates an active course of biosynthetic processes aimed at forming the muscle fiber structure. Connective tissue in this period of life is abundantly represented in the muscle, while the endomysium forms thin layers, and the paratenon, on the contrary, very wide layers of loose connective tissue (Figure 1).

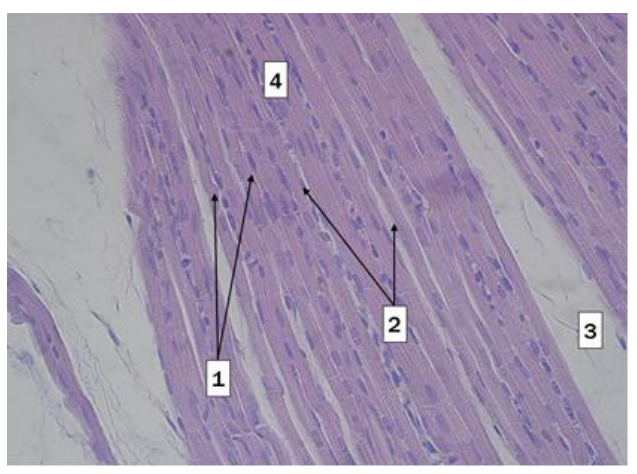

Fig. 1. Longitudinal section of the quadriceps femoris muscle in a day old chicken of cross Smena 8 - Plymouthrock.

The transverse striation in the muscle fibers is clearly visible. 1 - muscle fibers,

2 - endomysium, 3 - perimysium,

4 - bundles of muscle fibers.

Staining with hematoxylin and eosin, ob. 40, oc. 10 .

From 1 to 50 days (Figure 2) of chicken growth and development during muscle development, the following trends are observed: the area of muscle tissue, the diameter of muscle fibers and their bundles increase, the thickness of the endomysium does not change, the thickness of the perimysium decreases (Table 1).

Table 1. Morphometric characteristics of the quadriceps femoris and the superficial pectoral muscle of chickens of hens of Smena 8 "Plymouthrock" cross of meat and egg productivity direction

\begin{tabular}{|l|c|c|}
\hline \multicolumn{3}{|c|}{ 1st day after hatching } \\
\hline Indicators & $\begin{array}{c}\text { Quadriceps } \\
\text { femoris }\end{array}$ & $\begin{array}{c}\text { Superficial pectoral } \\
\text { muscle }\end{array}$ \\
\hline Area of muscle tissue, \%* & $75 \pm 1.45$ & $80 \pm 2.13$ \\
\hline Thickness of muscle fibers, microns & $7.68 \pm 0.54$ & $6.81 \pm 0.43$ \\
\hline Thickness of muscle fiber bundles, microns & $82 \pm 7.84$ & $78 \pm 6.76$ \\
\hline Endomysium thickness, microns & $4.43 \pm 0.23$ & $3.28 \pm 0.76$ \\
\hline Perimysium thickness, microns & $30.7 \pm 3.15$ & $20.2 \pm 2.41$ \\
\hline Number of fibers per HPF** & $13 \pm 1$ & $15 \pm 1$ \\
\hline \multicolumn{2}{|c|}{ 8th day after hatching } \\
\hline Area of muscle tissue, \%* & $79 \pm 2.26$ & $84 \pm 2.51$ \\
\hline Thickness of muscle fibers, microns & $10.91 \pm 0.23$ & $8.22 \pm 0.53$ \\
\hline Thickness of muscle fiber bundles, microns & $112 \pm 7.12$ & $121 \pm 8.44$ \\
\hline Endomysium thickness, microns & $3.87 \pm 0.78$ & $3.36 \pm 1.12$ \\
\hline Perimysium thickness, microns & $29.1 \pm 5.13$ & $24.2 \pm 3.56$ \\
\hline Number of fibers per HPF** & $15 \pm 1$ & $17 \pm 1$ \\
\hline \multicolumn{2}{|c|}{ 20th day after hatching } \\
\hline Area of muscle tissue, \%* & $81 \pm 2.83$ & $87 \pm 2.14$ \\
\hline Thickness of muscle fibers, microns & $13.1 \pm 0.23$ & $11.2 \pm 0.21$ \\
\hline Thickness of muscle fiber bundles, microns & $132 \pm 6.43$ & $144 \pm 7.5$ \\
\hline
\end{tabular}




\begin{tabular}{|l|c|c|}
\hline Endomysium thickness, microns & $3.1 \pm 0.56$ & $2.95 \pm 0.66$ \\
\hline Perimysium thickness, microns & $31.5 \pm 1.57$ & $24.2 \pm 6.13$ \\
\hline Number of fibers per HPF** & $14 \pm 1$ & $16 \pm 1$ \\
\hline \multicolumn{2}{|c|}{ 29th day after hatching } \\
\hline Area of muscle tissue, \%* & $85 \pm 2.61$ & $91 \pm 2.15$ \\
\hline Thickness of muscle fibers, microns & $14.7 \pm 0.42$ & $12.75 \pm 0.23$ \\
\hline Thickness of muscle fiber bundles, microns & $164 \pm 7.1$ & $171 \pm 6.34$ \\
\hline Endomysium thickness, microns & $3.24 \pm 0.12$ & $3.01 \pm 0.6$ \\
\hline Perimysium thickness, microns & $28 \pm 2.54$ & $23 \pm 1.43$ \\
\hline Number of fibers per HPF** $12 \pm 1$ & $14 \pm 1$ \\
\hline \multicolumn{2}{|c|}{ 50th day after hatching } \\
\hline Area of muscle tissue, \%* & $89 \pm 1.93$ & $92 \pm 2.06$ \\
\hline Thickness of muscle fibers, microns & $36.2 \pm 3.1$ & $27.9 \pm 1.77$ \\
\hline Thickness of muscle fiber bundles, microns & $292 \pm 14.1$ & $305 \pm 8.2$ \\
\hline Endomysium thickness, microns & $4.7 \pm 1.1$ & $3.96 \pm 0.13$ \\
\hline Perimysium thickness, microns & $21 \pm 3.44$ & $17.5 \pm 2.4$ \\
\hline Number of fibers per HPF** & $5 \pm 1$ & $6 \pm 1$ \\
\hline
\end{tabular}

* comparative morphometric analysis was performed at a magnification 400 .

**calculation was carried out in the standard HPF of the microscope at a magnification 1000.

The morphometric analysis of the muscle tissue parameters of a day-old hen chicken showed that the area of the muscle tissue and the number of muscle fibers are larger in the SPM, and the diameter of the muscle fiber is smaller compared to the QFM. This feature persists in the subsequent periods of hen development, and the most significant increase in the diameter of the muscle fiber is observed in the period from 29 to 50 days of development, which can be considered a critical stage in hen myohistogenesis.

Based on the analysis of the digital material, it can be noted that from the 1 st to the 50 th day of development, the area of muscle tissue increases in QFM by $18.6 \%$, in SPM - by $15 \%$, the diameter of muscle fibers increases (in QFM by 4.7 times, in SPM by 4.1 times) and the diameter of muscle fiber bundles (in QFM by 3.5 times, in SPM by 3.9 times). Against this background, the thickness of the perimysium decreases in QFM by $30 \%$, in SPM by $12.5 \%$.

\subsection{Histological and morphometric characteristics of skeletal muscles of the Manchurian golden quail}

To identify the general patterns and specific features of myogenesis, similar studies were conducted on the material obtained from quails. The muscles of quail chickens by the 1 st day after hatching appear to be fully formed: the muscle fibers have a pronounced transverse striation, they form bundles. The connective tissue component of muscle forms thin layers of endomysium and quite powerful ones layers - perimysium (Figure 3).

By the 42nd day, the thickness of both the muscle fiber itself and its bundles, as well as endo- and perimysium, increases. Light-optical studies have shown the presence of numerous neurovascular bundles (Figures 4, 5,6) located in the perimysium. In Mallory staining, there are differences in the basophily of muscle fibers, reflecting their heterogeneity and belonging to different types. 


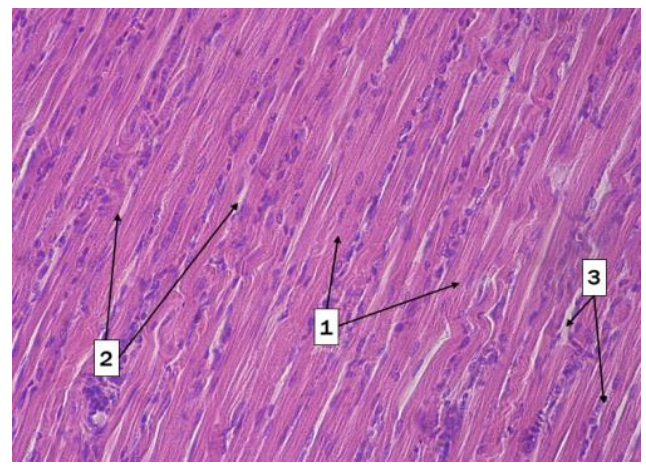

Fig. 3. Longitudinal section of the quadriceps femoris in a day-old Manchurian golden quail chicken. The transverse striation of the muscle fibers is clearly visible. 1 - muscle fibers, 2 endomysium, 3 - perimysium.

Staining with hematoxylin and eosin, ob. 40, oc. 10.

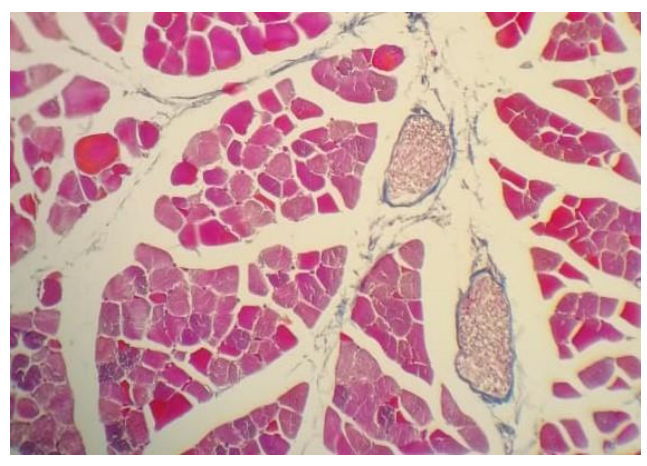

Fig. 5. Bundles of muscle fibers and bundles of nerve fibers between them in the superficial pectoral muscle of the Manchurian golden quail on the 50th day after hatching.

Mallory staining, ob. 40 , oc. 10 .

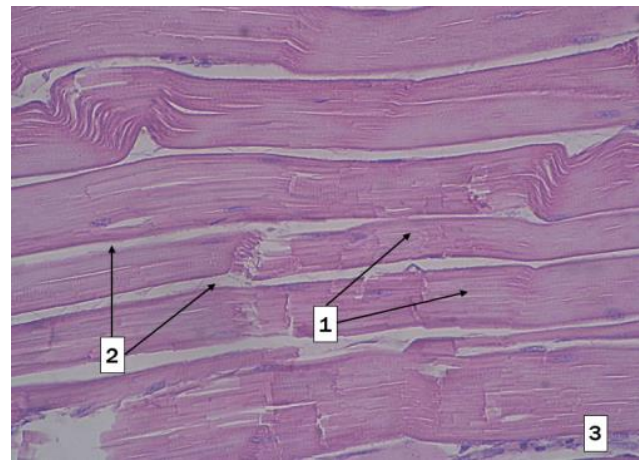

Fig. 4. Longitudinal section of the quadriceps femoris in a 42-day-old Manchurian golden quail chicken. The transverse striation of the muscle fibers is clearly visible. 1 - muscle fibers, 2 endomysium, 3 - perimysium.

Staining with hematoxylin and eosin, ob. 40, oc. 10.

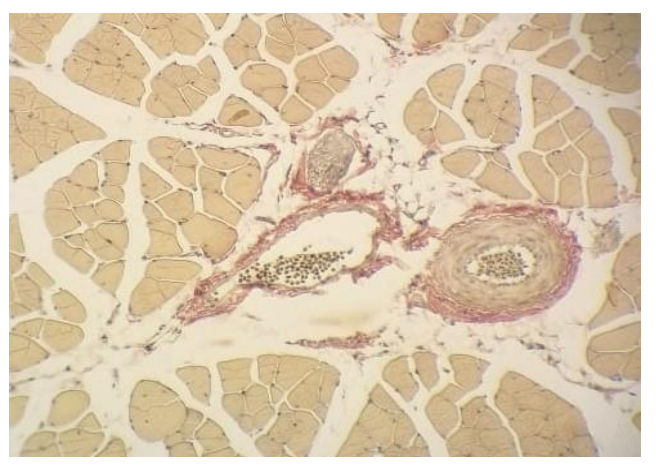

Fig. 6. Muscle fibers in the superficial pectoral muscle and the neurovascular bundle between them in the Manchurian golden quail on the 50th day after hatching. Cross-section.

Van Gieson staining, ob. 40, oc. 10.

Morphometric studies found that at all observation periods, the area of muscle tissue, the diameter of muscle fibers and their bundles in quails, in contrast to hens, is greater in QFM, and the number of muscle fibers - in SPM (Table 2).

Table 2. Morphometric parameters of the quadriceps femoris and superficial pectoral muscles in chickens and adult quails of the Manchurian golden breed

\begin{tabular}{|l|c|c|}
\hline \multicolumn{3}{|c|}{ 1st day after hatching } \\
\hline \multicolumn{1}{|c|}{ Indicators } & $\begin{array}{c}\text { Musculus quadriceps } \\
\text { femoris }\end{array}$ & $\begin{array}{c}\text { Superficial pectoral } \\
\text { muscle }\end{array}$ \\
\hline Area of muscle tissue, \%* & $78 \pm 3$ & $74 \pm 2$ \\
\hline Thickness of muscle fibers, microns & $4.19 \pm 0.32$ & $4.28 \pm 0.52$ \\
\hline Thickness of muscle fiber bundles, microns & $66.1 \pm 4.54$ & $43.1 \pm 4.65$ \\
\hline Endomysium thickness, microns & $2.12 \pm 0.18$ & $1.76 \pm 0.54$ \\
\hline Perimysium thickness, microns & $8.47 \pm 2.65$ & $7.89 \pm 7.27$ \\
\hline Number of fibers per HPF** & $22 \pm 2$ & $23 \pm 3$ \\
\hline
\end{tabular}




\begin{tabular}{|c|c|c|}
\hline \multicolumn{3}{|c|}{ 8th day after hatching } \\
\hline Area of muscle tissue, $\% *$ & $84 \pm 3.21$ & $82 \pm 2.14$ \\
\hline Thickness of muscle fibers, microns & $7.98 \pm 0.31$ & $6.84 \pm 1.18$ \\
\hline Thickness of muscle fiber bundles, microns & $88.7 \pm 7.65$ & $70.1 \pm 11.3$ \\
\hline Endomysium thickness, microns & $3.59 \pm 0.83$ & $1.88 \pm 2.01$ \\
\hline Perimysium thickness, microns & $8.02 \pm 2.12$ & $5.82 \pm 3.15$ \\
\hline Number of fibers per $\mathrm{HPF}^{* *}$ & $13 \pm 1$ & $14 \pm 1$ \\
\hline \multicolumn{3}{|c|}{ 21st day after hatching } \\
\hline Area of muscle tissue, $\% *$ & $85 \pm 2$ & $82 \pm 3$ \\
\hline Thickness of muscle fibers, microns & $10.1 \pm 0.23$ & $9.04 \pm 0.41$ \\
\hline Thickness of muscle fiber bundles, microns & $144 \pm 5.12$ & $159 \pm 3.65$ \\
\hline Endomysium thickness, microns & $4.15 \pm 1.24$ & $3.05 \pm 1.51$ \\
\hline Perimysium thickness, microns & $15.3 \pm 3.21$ & $11.1 \pm 2.04$ \\
\hline Number of fibers per HPF** & $15 \pm 1$ & $16 \pm 2$ \\
\hline \multicolumn{3}{|c|}{ 35th day after hatching } \\
\hline Area of muscle tissue, $\% *$ & $87 \pm 2$ & $85 \pm 2$ \\
\hline Thickness of muscle fibers, microns & $12.6 \pm 1.23$ & $10.6 \pm 2.04$ \\
\hline Thickness of muscle fiber bundles, microns & $151 \pm 4.41$ & $162 \pm 4.12$ \\
\hline Endomysium thickness, microns & $2.18 \pm 0.34$ & $2.68 \pm 1.63$ \\
\hline Perimysium thickness, microns & $26.4 \pm 3.38$ & $21.1 \pm 3.24$ \\
\hline Number of fibers per $\mathrm{HPF}^{* *}$ & $14 \pm 2$ & $12 \pm 2$ \\
\hline \multicolumn{3}{|c|}{ 42nd day of the hatching } \\
\hline Area of muscle tissue, $\% *$ & $88 \pm 4$ & $86 \pm 3$ \\
\hline Thickness of muscle fibers, microns & $21.7 \pm 1.24$ & $18.3 \pm 1.05$ \\
\hline Thickness of muscle fiber bundles, microns & $169 \pm 5.13$ & $189 \pm 5.32$ \\
\hline Endomysium thickness, microns & $3.28 \pm 1.42$ & $2.52 \pm 1.42$ \\
\hline Perimysium thickness, microns & $21.5 \pm 3.15$ & $23.2 \pm 3.32$ \\
\hline Number of fibers per HPF** & $12 \pm 1$ & $13 \pm 2$ \\
\hline
\end{tabular}

* comparative morphometric analysis was performed at a magnification 400.

**calculations were carried out in the standard HPF of the microscope at a magnification 1000.

According to the data obtained, the general trends in the formation of the structure of the studied quail muscles are as follows. From day 1 to day 42 of development, the area of muscle tissue increases in QFM by 12\%, in SPM - by 16\% (in chickens, on the contrary, more active growth was noted in QFM), the diameter of muscle fibers increases (in QFM by 5 times, in SPM by 4.2 times) and the diameter of muscle fiber bundles (in QFM by 2.6 times, in SPM by 4.4 times). At the same time, the perimysium thickness, in contrast to hens, does not decrease, but increases in QFM by 2.5 times, and in SPM - by 2.8 times.

\subsection{Results of electronic microscopic studies of the muscles of the Manchurian golden quail on the 50th day after hatching}

The muscles in quails on the 50th day after hatching are a highly differentiated structure of muscle fibers, in which the transverse striation is clearly defined, due to the developed contractile apparatus in the form of myofibrillas consisting of sarcomeres (Figure 7). The muscle fibers contain many nuclei, which can be located both on the periphery under the sarcolemma, and in the fiber thickness among the myofibrillas. The nuclei are 
morphofunctionally active, contain 2-3 nucleoli and an abundance of RNP granules in the karyoplasm, which ensures a high level of metabolism of the protein synthesis apparatus in the fiber (Figure 8). In some fibers, nuclei with a strongly altered shape were observed, which allows to intensify the metabolism between nucleus and cytoplasm. It is noteworthy that the Z-lines stretch through all the fiber myofibrillas. There are the cisterns of the sarcoplasmic reticulum, mitochondria, and glycogen grains between the myofibrillas. Glycogen grains are better represented in the SPM of quail and are often found near the Zlines. In SPM, a larger diameter of myofibrillas was noted, compared with QFM, as well as separation from each other due to wide layers of sarcoplasm. Mitochondria are an important component of muscle fibers. In the SPM of quails, they are small, round, oval or irregular in shape, located at a distance from each other, as well as at a distance from the myofibrillas. Blood capillaries are often found between the muscle fibers (Figure 7).

In QFM, myofibrillas are narrow and form bundles of 3-4 elements located close to each other (Figure 8). Between these bundles there are wide layers of sarcoplasm, which contain large rod-shaped mitochondria, the length of which corresponds to the length of 5-6 sarcomeres. Thus, the most pronounced differences in muscle tissue in SPM and QFM are manifested in the distribution of myofibrillas, as well as the size, number and localization of mitochondria in the fiber. In QFM, the muscle fibers are red, and they are characterized by the presence of peculiar mitochondrial complexes under the sarcolemma (Figure 9). Another feature of the ultrastructure of quail QFM is the presence of clearly visible cisterns of the sarcoplasmic reticulum, which forms groups of 3-5 rounded vesicles near the Z-lines of the myofibrilla.

In both muscles, small destructive changes in individual mitochondria were observed in the form of matrix enlightenment and cristae length shortening. We believe that these changes are due to structural transformations of muscle fibers. Between the muscle fibers in the connective tissue framework there is a large number of hemocapillaries, in the structure of which the nuclear apparatus is detected, with a predominance of euchromatin, ribonucleoproteins and nucleoli.

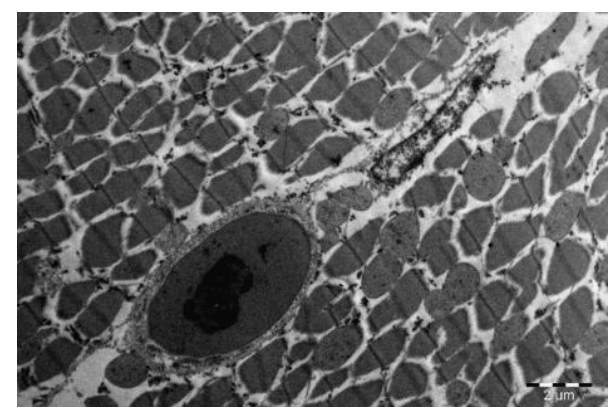

Fig. 7. Ultrastructural design of the superficial pectoral muscle in quail on the 50th day after hatching. The blood capillary is located between the muscle fibers.

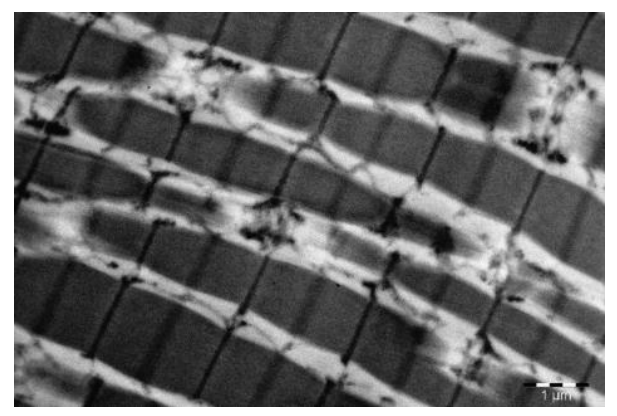

Fig. 8. Ultrastructural organization of the superficial pectoral muscle in quail on the 50th day after hatching. In the muscle fiber between the myofibrillas, there are wide layers of sarcoplasm. 


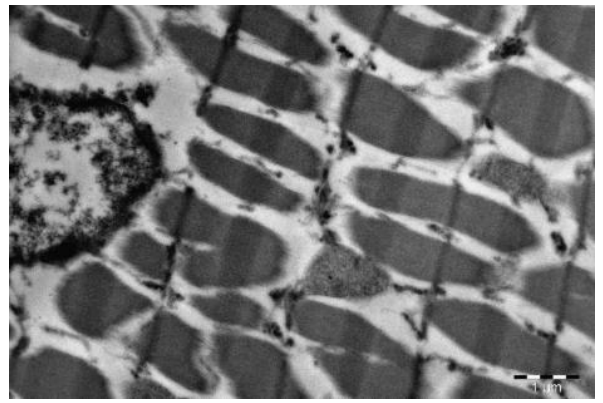

Fig. 9. Ultrastructural organization of the superficial pectoral muscle in quail on the 50th day after hatching. Euchromatin and RNP granules are observed in the nucleus. Mitochondria of oval or irregular shape are located in the central part of the fiber.

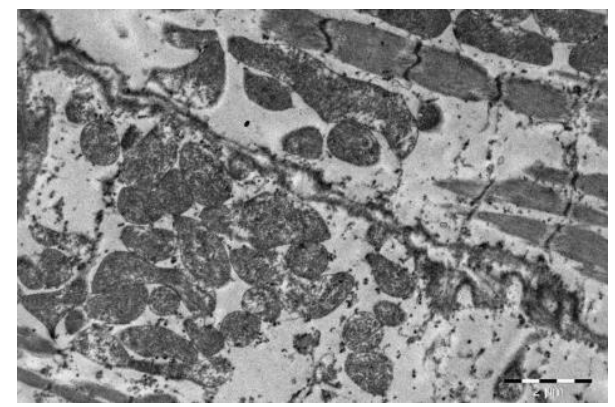

Fig. 11. Ultrastructural organization of the quadriceps femoris muscle in quail on the 50th day after hatching. The mitochondrial complex is located on the periphery of the fiber.

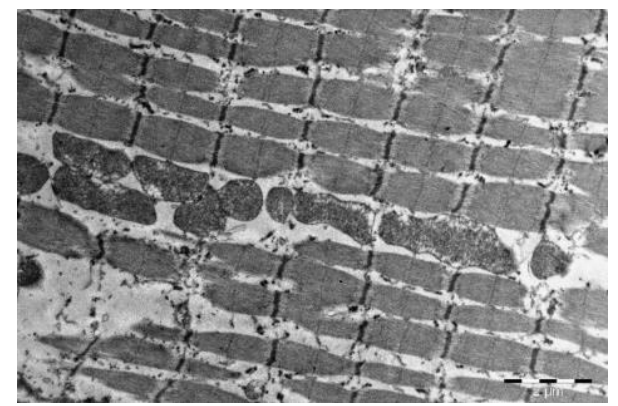

Fig. 10. Ultrastructural organization of the quadriceps femoris muscle in quail on the 50th day after hatching. In myofibrillas, transverse striation is visible. Large mitochondria are located in the central part of the fiber.

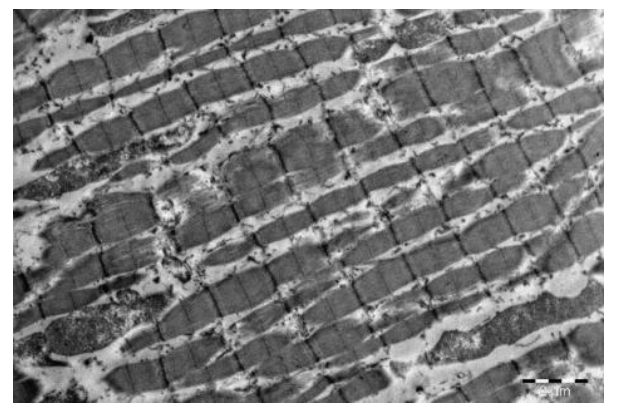

Fig. 12. Ultrastructural organization of the quadriceps femoris muscle in quail on the 50th day after hatching. In the muscle fiber, myofibrillas are narrow, located close to each other.

\section{Conclusions}

Thus, a comparative morphological and morphometric assessment of the studied skeletal muscles of hens and quails allowed to establish general patterns of their growth. Representatives of both groups of birds studied with an increase in the area of muscle tissue, the diameter of muscle fibers and their bundles, and the percentage of increase in these indicators is very similar. The difference is a more significant development of QFM in hens, and SPM - in quails. It is noteworthy that quails, unlike hens, had thickening of the endomysium and perimysium in both muscles.

On the basis of the conducted studies, we conducted a comprehensive study of the superficial pectoral muscle and quadriceps in hens of the Smena 8 "Plymouthrock" crosses and in quail of the Manchurian golden breed at the early stages of bird ontogenesis. The study of morphometric indicators showed that the general pattern is an increase in the area of muscle tissue and the diameter of muscle fibers with the age of chickens and quails, while simultaneously reducing their number in the field of view, as well as a decrease in the ratio between muscle and connective tissues. The quail has a higher level of vascularization and innervation in the studied muscles compared to the hen muscles. In the muscles of birds, the contractile apparatus is characterized by a strictly ordered structure. 
Ultrastructural analysis of SPM and QFM in adult quails showed that muscle fibers contain the largest representation of myofibrillas and mitochondria, which are characterized by close morphofunctional connections. At the same time, differences in the structural arrangement of myofibrillas, the location and shape of mitochondria, and the cisterns of the sarcoplasmic reticulum were revealed between QFM and SPM.

\section{References}

1. A.N. Belogurov, Morphofunctional adaptation of the internal organs of the Japanese quail in technological traumatism in industrial poultry farming (experimental and clinical studies), autoref. dis ... dr. vet. sc., 50 (M., 2013)

2. V.F. Vrakin, M.V. Sidorova, Anatomy and histology of poultry, 426 (Moscow, KolosS, 2001)

3. P.A. Glagolev, Bulletin of TAA, 4, 155-170 (1959)

4. R.K. Danilov, Differentiation of myosatellitocytes and muscle fibers in embryogenesis and reparative histogenesis, Dis. doc. med. sc. (Kuibyshev, 1982)

5. R.K. Danilov, Essays on the histology of muscle tissues 50 (Ufa, Bashkortostan, 1994)

6. V.I. Ippolitova, K.S. Zablotskaya, TAA reports, 164, 146-152 (1970)

7. G.V. Kondratov, E.N. Borkhunova, Morphology, 3, 40 (2016)

8. G.V. Kondratov, Features of skeletal muscle histogenesis in chickens of different productivity trends, Autoref. diss ... c. b. sc., 26 (M., 2016)

9. I.I. Kochish, N.A. Slesarenko, L.P. Troyanovskaya, A.N. Belogurov, Quail farming: problems and ways to solve them, 157 (M., 2015)

10. T.V. Chernysheva, Age-related structure features of organs of movement in chickens: autoref, Dis ... cand. biol. sc. Moscow, TAA, 15 (1974).

11. G.R. Shakirova, N.A. Mufazalova, S.M. Shakirova, Success of modern natural studies, 2, 20-21 (2009)

12. G.R. Shakirova, V.A. Bolshunov, S.M. Shakirova, Issues of regulatory and legal regulation in veterinary medicine, 2, 131-134 (2019)

13. Yu.F. Yudichev, G.I. Barabanshchikova, Questions of morphology, physiology and nutrition of farm animals and fur-bearing animals: Scientific works of the Omsk vet. Institute, 35 (1), 3-9 (1978)

14. M.D. Shmerling, E.E. Filyushina, I.I. Buzuyeva et al., Skeletal muscle. Structural and functional aspects of adaptation, 121 (Novosibirsk: Nauka, Siberian Branch, 1991)

15. B.R. Eisenberg, / J. Ultrastruct, 54, 76-88 (1976)

16. G.F. Gauthier, H.A. Padycyla, Ibid, 28 (2), 333-354 (1966)

17. M. Luxey, B. Berki, W. Heusermann, S. Fischer, P. Tschopp, Developmental Biology, 458 (2), 133-140 (2020)

18. G. Schippel, K. Schippel, K. Welt, Anat. Ans., 140 (4), 400-404 (1976)

19. J.R. Torrella, V. Fouces, J. Palomeque, G. Viscor, Journal of Anatomy, 192 (2), 211222 (1998)

20. S. Webb, J. Pathol., 106 (4), 221-228 (1972) 